\title{
CRITICAL REVIEWS ON HOLOGRAPHIC MEASURE OVER THE MULTIVERSE
}

\author{
SUNGWOOK E. HONG \\ Department of Physics, KAIST, Daejeon 305-701, South Korea \\ innocent@muon.kaist.ac.kr \\ DONG-HAN YEOM \\ Department of Physics, KAIST, Daejeon 305-701, South Korea \\ eostm@muon.kaist.ac.kr \\ HEESEUNG ZOE \\ Department of Physics, KAIST, Daejeon 305-701, South Korea \\ hszoe@muon.kaist.ac.kr
}

\begin{abstract}
In this talk, the holographic measure based on black hole complementarity is critically reviewed. By noticing the similarities between the causal structure of an inflationary dS space and that of a black hole, we have considered the complementarity principle between the inside and the outside of the causal horizon as an attractive way to count the inflationary multiverse. Even though the holographic measure relieves the Boltzmann brain problem and stresses the physical reality based on observations, it could be challenged by the construction of counterexamples, both on regular black holes and charged black holes, to black hole complementarity.
\end{abstract}

Keywords: Inflationary multiverse; holographic measure; complementarity principle.

\section{Introduction}

If the paradigm of inflation is correct, then there would be no way other than concluding the global structure is the multiverse, a complex mixture of thermalized and inflating regions. In the context of the multiverse, we could explore the cosmic finetuning problem by asking statistical distributions over the multiverse. The approach based on the statical distribution is deeply related to anthropic reasoning. Whether our universe is typical among the possible pocket universes would be a important signature on the statistical inference and the reasoning of quantum cosmology.

In general, the complexity of the global structure of the multiverse depends on the shape of inflation potentials. However, Guth pointed out that the eternal inflation could be activated even with a simple potential, and there would be no proper way to quantify the statistical distribution based on the volume fractions due to the infinity and the slice-dependence. ${ }^{1}$ Thus it implies that we may fail to 
handle the complexity and to specify the measure over the inflationary multiverse. This problem is called as 'measure problem'.

Among many proposals to overcome the measure problem, there have been a remarkable category of attempts inspired by the similarities between physical properties (e.g. causal structures, thermodynamics, etc.) of black holes and those of de Sitter (dS) spaces. Susskind proposed that the complementarity principle should be involved in specifying the measure from his speculations for resolving the black hole information paradox..$^{2,3}$ In the line of this thought, Bousso has developed holographic measure based on black hole complementarity. ${ }^{4}$

\section{Black Hole Complementarity and Holographic Measure}

\subsection{Black hole complementarity}

According to black hole complementarity, there is no consistent way of describing both the inside and the outside of the black hole horizon. ${ }^{3}$ If we take a free-falling observer who steps into the horizon, he would investigate the internal structure of the black hole and confirm the original information which we used to make the black hole. If we take an additional asymptotic observer who stays outside of the horizon, he can reconstruct the original information from Hawking radiations by considering correlations among them. From a heuristic argument using the statistical average by tracing out the black hole internal states, Page suggested that Hawking radiation begins to carry the information only after so called "information retention time", which is the moment when the size of the black hole horizon decreases into the half. ${ }^{5}$
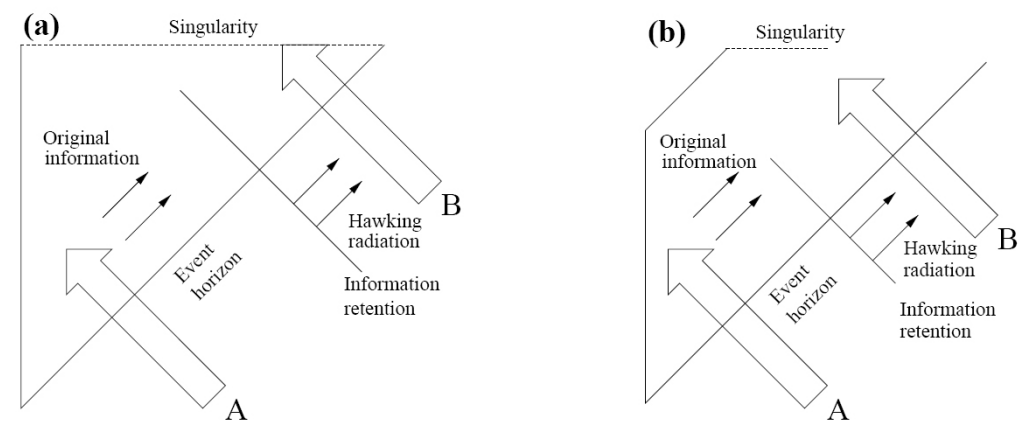

Fig. 1. Possibilities of the duplication experiments in different types of black holes: (a) duplication experiment is not possible in Schwartzschild black hole, due to the lack of time in the presence of a singularity inside the black hole. (b) it may be possible to find or construct a black hole geometry where we have enough time to do the duplication experiment.

Essentially, black hole complementarity assumes that the all information is duplicated on the stretched horizon. So now we have actually the duplication of quantum 
information, which is the obvious violation of quantum principles. However, these two observers cannot communicate by quanta whose energy is less than the total black hole mass energy, so this complementary viewpoint is logically safe and operationally meaningful. This is quite true at least in Schwarzschild black holes. Let us consider two observers A and B in Fig. 1(a). At first, send the observer A to investigate the inner states of the Schwarzschild black hole and identify the original information about the resources which made the black hole. After the information retention time of the order of $M^{3}$ (the black hole mass is $M$ ) send the observer $\mathrm{B}$ to check whether the information is really duplicated. We can calculate the time for the observer B to hit the singularity at $X_{-} X_{+}=M^{2}$ in the Kruskal coordinate, which is $M^{2} \exp \left(-M^{2}\right)$ : If the observer A send his information to observer $\mathrm{B}$, he could do it only by sending quanta whose energy is order of $M^{-2} \exp \left(M^{2}\right)$ which is much greater than the total mass energy. It means that the observer B can never confirm that the quantum information is duplicated.

\subsection{Holographic measure}

If the complementarity principle is a fundamental principle of quantum gravity, it should be considered even when we try to specify the measure over the inflationary multiverse, because the inflationary de Sitter universe has its own causal horizon. According to complementarity, there must be no way to involve the causal parts outside of the observer horizon in the physical description. This point makes the reasoning of holographic measure economic and plausible, which focuses on only the causally connected parts of the multiverse and which abandons the outside of the cosmic horizon.

There are two major merits in the holographic measure. First, the holographic measure makes the issues related with typicality simple: Since the complementarity restricts the discussion only within the cosmic horizon, the holographic measure can relieve Youngness paradox and Boltzmann brain problem which are evoked by considering the whole global structure of the multiverse. Second, the holographic measure makes the multiverse to be testable by observations in principle: Although the whole multiverse is still always beyond our ability of observation, the concept of multiverse can be tested and modified by observations within the observer horizon, since the complementarity, as a fundamental principle, protects the logic that neglects magnificent parts of the global structure.

\section{Looking for Counterexamples to Complementarity}

In Fig. 1(a), the main reason that black hole complementarity is working in a Schwarzschild black hole is the lack of time to check whether the information is duplicated and so no-cloning theorem is violated. Therefore we can ask whether we can find or generate black hole geometries to have enough time to do the duplication experiment. The authors claim that we can find such counterexamples to black hole complementarity and they can also challenge the holographic measure based on it. 


\subsection{Charged black holes}

The evolution of a charged black hole is dynamically described in Ref. 6. We start from complex massless scalar fields coupled with the electromagnetic field. We can include Hawking radiation effectively by considering the renormalized tensor and get the Penrose diagram as the result of numerical simulations (see the left panel of Fig. 2). There exists a singular region over the Cauchy horizon, and the region near the inner horizon due to mass inflation also seems to be singular. However, by increasing the number of massless scalar fields, we can obtain the regular region between the inner horizon and the Cauchy horizon. It means that we can have enough time to do the duplication experiment and so black hole complementarity is not correct in this charged black hole any longer.

Still there is a loophole in the argument: If there is a non-local effect in Hawking radiation, as Horowitz and Maldacena proposed, then the duplication experiment may not be possible, because Hawking radiation can carry the information only after the matter states is projected at the singularity near the Cauchy horizon. ${ }^{7}$ However, if we consider randomized mixing of quantum states on the stretched horizon, we can get rid of this loophole. After Hawking radiation comes out from a charged black hole, if we add one bit of quantum information, then it will come out as Hawking radiations in the time of order $M \ln M$. Thus right after this charged black hole acts like an information mirror, the duplication experiment is possible. ${ }^{8}$
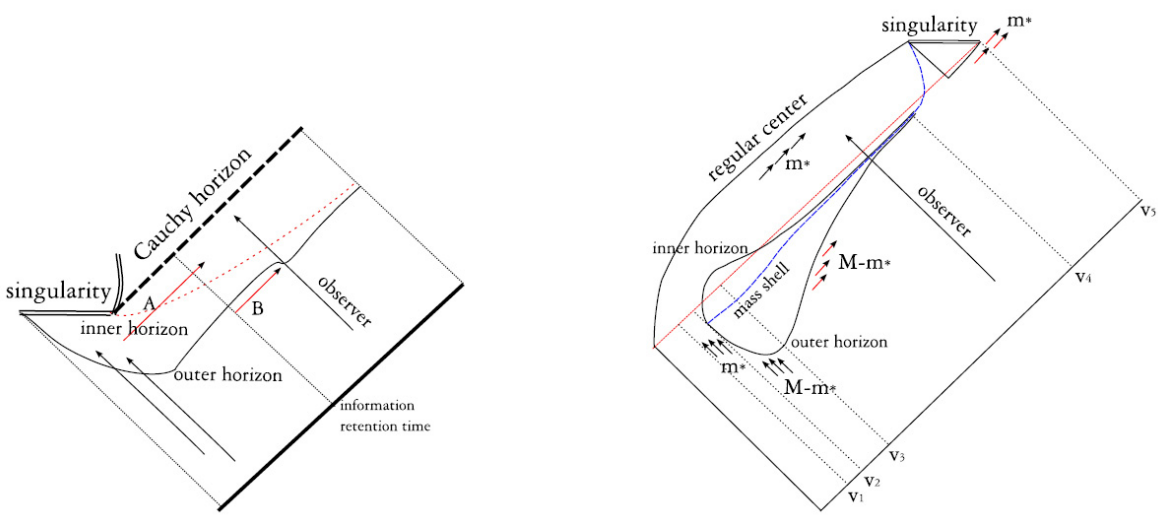

Fig. 2. Causal structures of a dynamical charged black hole (left) and Frolov, Markov, and Mukhanov's regular black hole (right).

\subsection{Regular black holes}

It is possible to construct a counterexample with regular black holes whose inside structure is a de Sitter space but the outside is a Schwarzschild black hole (see the right panel of Fig. 2). ${ }^{9}$ The cosmological constant of the de Sitter space is 
coupled with a scalar field, which decides the dynamical evolution of the regular black hole. The scalar field tunnels onto the greater vacuum energy and then the regular black hole goes to the extreme limit. (It means that the tunneling events produce statistical ensembles and some of them may satisfy the criteria for the duplication experiment.) In this case, we have enough time to do the duplication experiment between the information retention time and, surely, the extreme limit.

\subsection{Inflationary de Sitter spaces}

By the previous counterexamples, black hole complementarity seems to be invalid in general, which shows that the holographic measure has unstable ground for its consistency. Up to the authors' knowledge, complementarity in de Sitter spaces is more difficult to be realized than in black holes, since even some physical concepts like S-matrix are not well-defined in de Sitter spaces.

However, it is also difficult to invalidate dS complementarity directly by constructing counterexamples where we do the duplication experiment: The information retention time of de Sitter space is estimated as much as Poincare recurrence time. ${ }^{10}$ It means that if we want to observe dS (Hawking) radiation with information, we should wait for the whole lifetime of dS space. This may be a good news to the holographic measure, for it would not get troubled with counterexamples using duplication experiments.

\section{Conclusion}

Black hole complementarity is a fascinating idea to resolve the black hole information puzzle. The holographic measure based on the black hole complementarity has some nice features to the measure problem over the inflationary multiverse: to relieve the issues around the typicality and to relate the discussions directly to observational knowledge. However, black hole complementarity seems to be invalidated by counterexamples permitting duplication experiments in charged black holes and regular black holes, which affect critically on the consistency of the holographic measure.

\section{References}

1. A. H. Guth, Inflation and Eternal Inflation, Phys. Rept. 333, 555 (2000) [arXiv:astro$\mathrm{ph} / 0002156]$.

2. L. Susskind, The Anthropic Landscape of String Theory, arXiv:hep-th/0302219.

3. L. Susskind, L. Thorlacius and J. Uglum, The Stretched Horizon and Black Hole Complementarity, Phys. Rev. D 48, 3743 (1993) [arXiv:hep-th/9306069]; L. Susskind, String Theory and the Principles of Black Hole Complementarity, Phys. Rev. Lett. 71, 2367 (1993) [arXiv:hep-th/9307168]; L. Susskind and L. Thorlacius, Gedanken Experiments Involving Black Holes, Phys. Rev. D 49, 966 (1994) [arXiv:hep-th/9308100].

4. R. Bousso, Holographic Probabilities in Eternal inflation, Phys. Rev. Lett. 97, 191302 (2006) [arXiv:hep-th/0605263]; R. Bousso and B. Freivogel, A Paradox in the Global Description of the Multiverse, JHEP 0706, 018 (2007) [arXiv:hep-th/0610132]. 
5. D. N. Page, Expected Entropy of a Subsystem, Phys. Rev. Lett. 71, 1291 (1993) [arXiv:gr-qc/9305007].

6. S. E. Hong, D. i. Hwang, E. D. Stewart and D. h. Yeom, The Causal Structure of Dynamical Charged Black Holes, arXiv:0808.1709 [gr-qc].

7. S. E. Hong, D. i. Hwang, D. h. Yeom and H. Zoe, Black Hole Complementarity with Local Horizons and Horowitz-Maldacena's Proposal, JHEP 0812, 080 (2008) [arXiv:0809.1480 [gr-qc]].

8. D. H. Yeom and H. Zoe, Black Hole Complementarity Gets Troubled with a Dynamical Charged Black Hole, arXiv:0811.1637 [gr-qc].

9. D. H. Yeom and H. Zoe, Constructing a Counterexample to the Black Hole Complementarity, Phys. Rev. D 78, 104008 (2008) [arXiv:0802.1625 [gr-qc]].

10. U. H. Danielsson, D. Domert and M. E. Olsson, Miracles and Complementarity in de Sitter Space, Phys. Rev. D 68, 083508 (2003) [arXiv:hep-th/0210198]; M. K. Parikh and J. P. van der Schaar, Not One Bit of de Sitter Information, JHEP 0809, 041 (2008) [arXiv:0804.0231 [hep-th]]. 